\section{One stage implant-based reconstruction of the breast in a single patient: comparison between mesh and modified dual plane technique}

\author{
Egidio Riggio, ${ }^{1}$ Joseph Ottolenghi, ${ }^{1}$ \\ Veronica Grassi, ${ }^{2}$ Maurizio Nava' \\ 1 Unità di Chirurgia Plastica e Ricostruttiva, \\ IRCCS Fondazione - Istituto Nazionale dei \\ Tumori, Milano, Italy; ${ }^{2}$ North West London \\ Hospitals NHS Trust, Northwick Park \\ Hospital, London, UK
}

\section{Abstract}

Acellular dermal matrix and similar meshes are used in breast reconstruction to cover the inferior implant pocket. We considered whether using a modified dual-plane technique in immediate breast reconstruction could improve the outcome when compared with mesh reconstruction. The paper presents a case of a patient who underwent one-stage bilateral immediate breast reconstruction (IBR) with $470 \mathrm{~g}$ implants, using alloplastic mesh in one side and the composed dual-plane pocket in the other. In the case described, the composed dual plane technique gave a better result in terms of absence of rippling and smoother surface at the palpation; the mesh coverage gave a better inframammary contour. Our case suggests that the modified dual-plane technique gives a satisfactory cosmetic outcome. It also reduces costs and avoids meshrelated complications. This technique should therefore be considered as an option to the mesh in implant-based breast reconstruction.

\section{Introduction}

The current standard for implant-based reconstruction after mastectomy is the twostage reconstruction with temporary expander, followed by permanent implant. In the first stage, a tissue expander is placed and expanded to the target volume in successive outpatient visits. In the second phase, the expander is exchanged with an implant and contralateral symmetry procedures, such as mastopexy, reduction, or augmentation, are performed.

The subpectoral implant insertion, though, is associated to some disadvantages, in particular the incomplete coverage of the prosthesis. This often requires delayed corrections to provide a good cosmetic result. A solution to this problem could be the use of newer meshes recently introduced on the market, which can give a more physiological shape providing better lower pole coverage. In particular, the acellular dermal matrix (ADM), incorporated into expander-based reconstruction, has been used by an increasing number of surgeons because it gives them the possibility to use larger fill volumes intraoperatively and to achieve a more aesthetically enhanced shape and appearance. This technique causes less tension on lower mastectomy skin flaps and better definition of the lateral and inframammary fold. It also leads to compartmentalization of the tissue expander with better support, eliminates the need for additional muscular dissection, and improves coverage and a better projection of lower pole. This permits a more natural appearance of the breast mound and improves the aesthetic outcome, yet being associated with lower rates of capsular contracture. The complication profiles appear to be similar to traditional tissue expander breast reconstruction; however, when used in a twostage procedure, $\mathrm{ADM}$ is more expensive and shows higher ratio of seroma (3.9), infection (2.7) and reconstructive failure (3.0\%). ${ }^{1}$

The introduction of ADMs to expander/implant breast reconstruction has gained worldwide popularity as it improves the surgeon's ability to obtain consistent positive results with an implant-based reconstruction.

Similarly, the immediate breast reconstruction (IBR) offers significant advantages, avoiding a second operation, reducing patients' discomfort and abating costs. Immediate breast reconstruction also reduces the duration of convalescence and offers an earlier restoration of body image and perception. ${ }^{2}$ Both skin-sparing and nipple-sparing mastectomy followed by IBR with autogenous tissue and/or silicone implants have recently become an attractive option for those women with breast cancer (BRCA) gene mutations looking for prophylactic mastectomies, and have hence been suggested as treatment options.

The advent of ADM is particularly useful for one stage implant placement as it provides a better lower pole expansion and facilitates the implant placement., ${ }^{3,4}$ In literature, there are few papers suggesting that the one-stage implant-based reconstruction with ADM, compared to the two-stage procedure conducted with expanders and ADM, has fewer complications and lower costs ${ }^{2,5}$ even adding the complications rate. ${ }^{6}$ Other papers describe the use of autologous techniques, such as the dual plane modified one, in order to avoid complications related to $\mathrm{ADM}^{7,8}$ while performing an immediate breast reconstruction.

This discordance in authors' view can partially explain why the one-stage reconstruction with ADM or similar materials is not yet recognized as a standard practice.

Our paper presents the case of a patient
Correspondence: Veronica Grassi, North West London Hospitals NHS Trust, Northwick Park Hospital, Watford Road, London, UK.

Tel.Fax: +44.788.522.5475.

E-mail: grassivero@gmail.com

Key words: immediate breast reconstruction, mesh, dual plane technique.

Contributions: ER, J0 and MN performed the surgical operations. ER reviewed the different versions of the paper. VG did the literature research and wrote the paper.

Conflict of interests: the authors declare no potential conflict of interests.

Received for publication: 14 May 2013.

Accepted for publication: 27 May 2013.

This work is licensed under a Creative Commons Attribution NonCommercial 3.0 License (CC BYNC 3.0).

(C) Copyright E. Riggio et al., 2013

Licensee PAGEPress, Italy

Surgical Techniques Development 2013; 3:e1 doi:10.4081/std.2013.e1

undergoing one-stage bilateral IBR using alloplastic mesh and a dual-plane pocket for the implant cover. This case is unique as it compares two different methods used to obtain the same immediate result.

\section{Case Report}

In January 2011, a 36-year-old woman, BRCA1 positive, underwent bilateral prophylactic nipple-sparing mastectomy and reconstruction using a mesh (Tiloop ${ }^{\circledR} ;$ GfE Medizintechnik GmbH, Nuremberg, Germany) in the left breast, and the modified dual-plane technique in the right breast to cover the silicone implants (Natrelle 410MF $470 \mathrm{~g}$; Allergan, Irvine, CA, USA). Informed consent was obtained from the patient.

The inframammary fold is marked, and a modified skin-sparing oblique lateral incision is performed. By directing more of the incision in an oblique to horizontal direction, avoiding the areolar border, the surgeon can have a better access to all quadrants of the breast, while minimizing the vertical skin defect and maximizing the excess skin available for expansion.

On the right breast, once the skin excess and flap thickness are assessed, the surgeon starts undermining the infero-lateral aspect of the serratus anterior muscle, and continues with the undermining of the pectoralis major. 
The lower border of the pocket must reach the inframammary fold. The muscle is partially cut medially, from the $4^{\text {th }}$ rib down to the inframammary limit where a complete release starts. Both the fibromuscular tissue and the deep fascia (musculofascial) are totally released by electocautery, along the inferior fold, producing a pocket extension of 1-2 cm. Then, the electrosurgical dissection of the superficial fascia (Scarpa) and related retinacula (Figure 1) can give further vertical extension of the lower pocket, $3-5 \mathrm{~cm}$ more. The technique can include 1-2 partial myotomies lying parallel and medial to the suture of the muscular access to the pocket, just in the lower-inner section of the muscle, with the aim of discharging the muscle strength in the portion of major projection of the implant. The anterior envelope of the implant pocket becomes dual, as composed of a stable and well-vascularized muscle layer at the upper two/thirds and skin-fat layer at the bottom third according to Riggio et al. ${ }^{9}$ (Figure 1).

On the left breast, the pectoralis major is disinserted. The lower section of the Tiloop ${ }^{\circledR}$ medium extralight $\left(16 \mathrm{~g} / \mathrm{m}^{2}\right)$ mesh is fixed to the inframammary fold using monofilament suture material, while its upper section is attached to the lateral and inferior edge of the muscle (Figure 2). Once the muscle and graft/dual plane interface is secured, two drains are placed each side. The pockets and the implants are rinsed with antibiotic irrigation during the procedure.

Drains were removed within day 13 , as less than $20 \mathrm{~mL}$ were collected. Routine perioperative antibiotic prophylaxis was given to the patient until the drains were removed. No immediate or late complications were reported by the patient and the degree of satisfaction on the cosmetic outcome was extremely high. The 12-month follow-up showed no contracture, Baker grade 1 (left) and 2 (right). On the left breast, there was rippling at the upper-inner quadrant and the device dots were easily palpable, probably due to a thinner layer of the tissue overlying the lower implant. The upper-pole skin thickness was nearly $1 \mathrm{~cm}$ bilaterally. The main difference was in the muscular hypotrophy of the left pectoralis major. The left inframammary contour was better defined but the breast resulted to be too much soft on palpation. The right breast instead showed a homogeneously smooth tissue coverage (Figure 3).

\section{Discussion}

Immediate implant reconstruction using ADM is considered to be cost-effective compared to the two-stage reconstruction.

As suggested by our case, we could perform the immediate implant coverage with the dual-

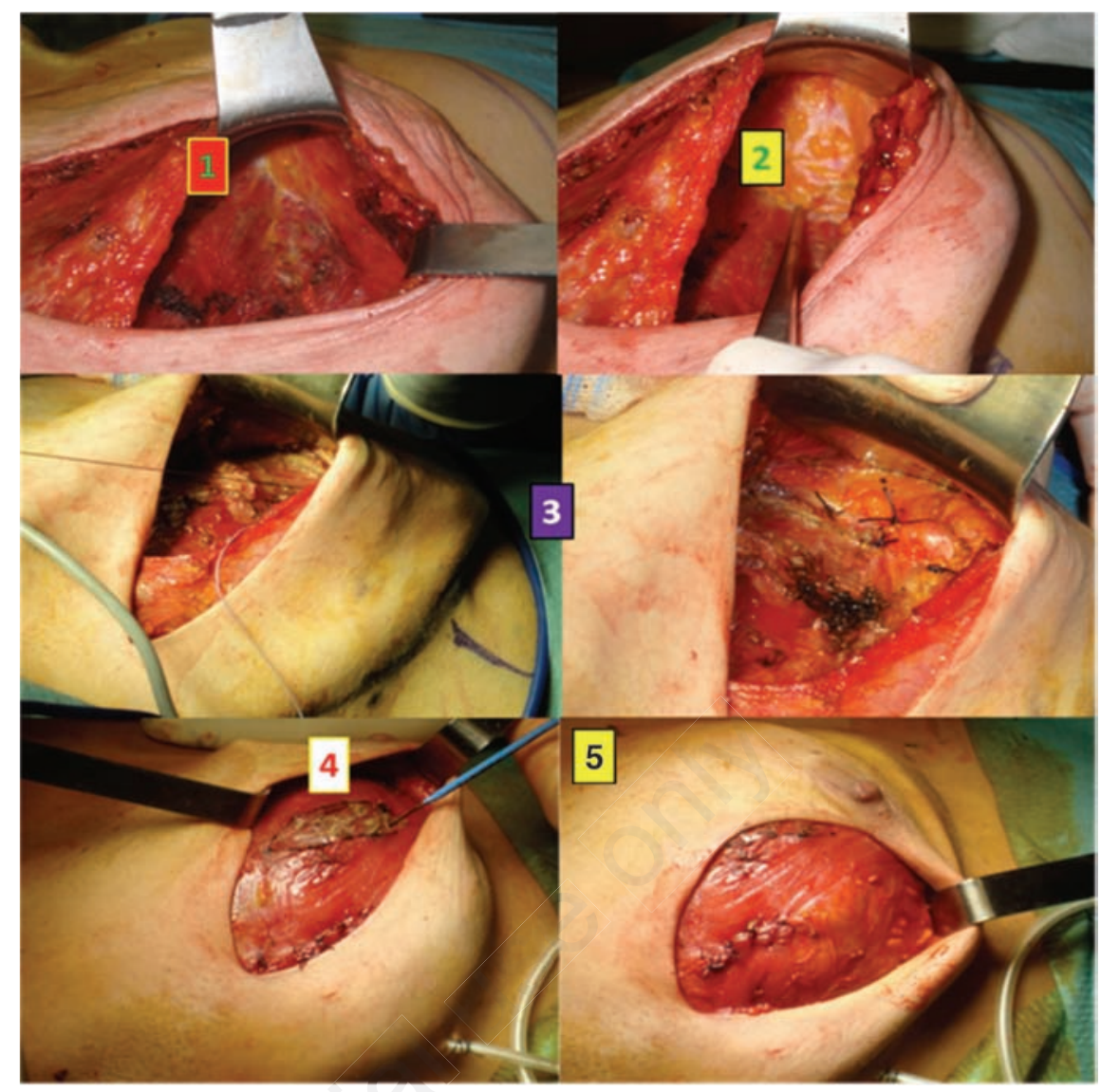

Figure 1. The dual-plane modified technique performed in the right breast. Both the fibromuscular tissue and the deep fascia are released at the inframammary fold, producing a pocket extension of $1-2 \mathrm{~cm}$ (1). The original full scoring of the superficial fascia and its attachments can gain further vertical extension of the lower pocket, $3-5 \mathrm{~cm}$ more (2). The technique can include a middle fixation of the fold using one-two vicryl-0 stitches in order to improve the submammary symmetry (3). One-two partial myotomies can be carried out medially to the muscular suture line (4). The implant coverage is not interrupted: muscle layer at the upper two/thirds and skin-fat layer at the bottom third (5).

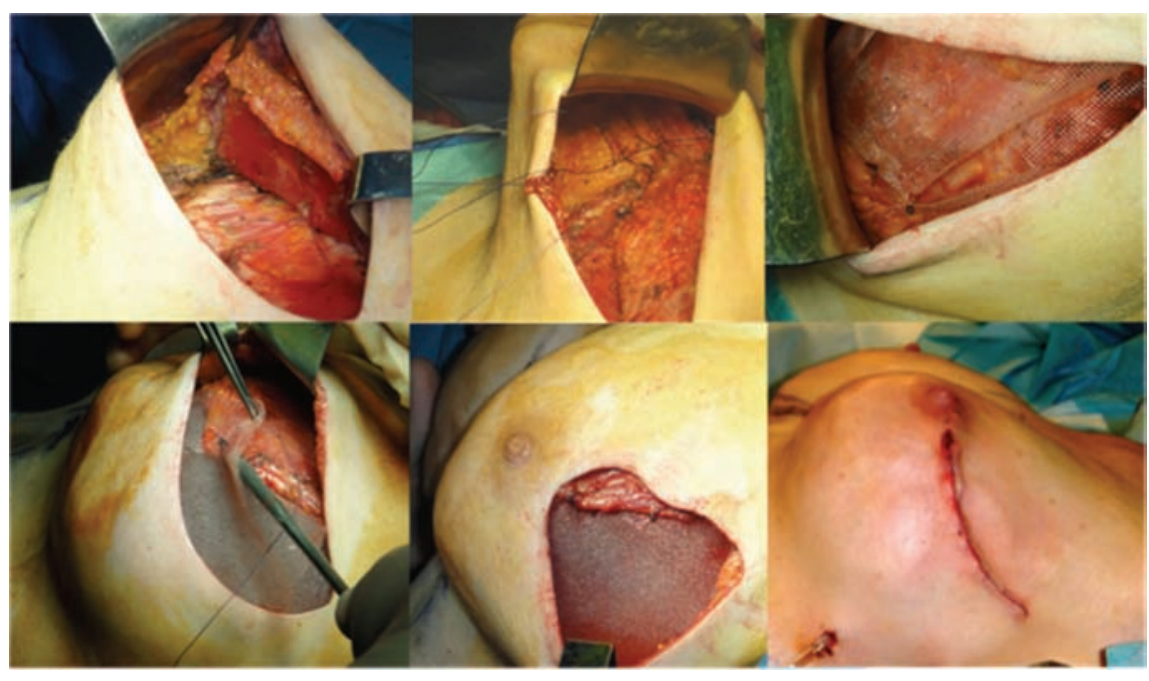

Figure 2. Preparation of the left breast pocket using a composite coverage made of pectoralis maior muscle and Tiloop ${ }^{\circledR}$ mesh placed along the inferior and lateral portions. 


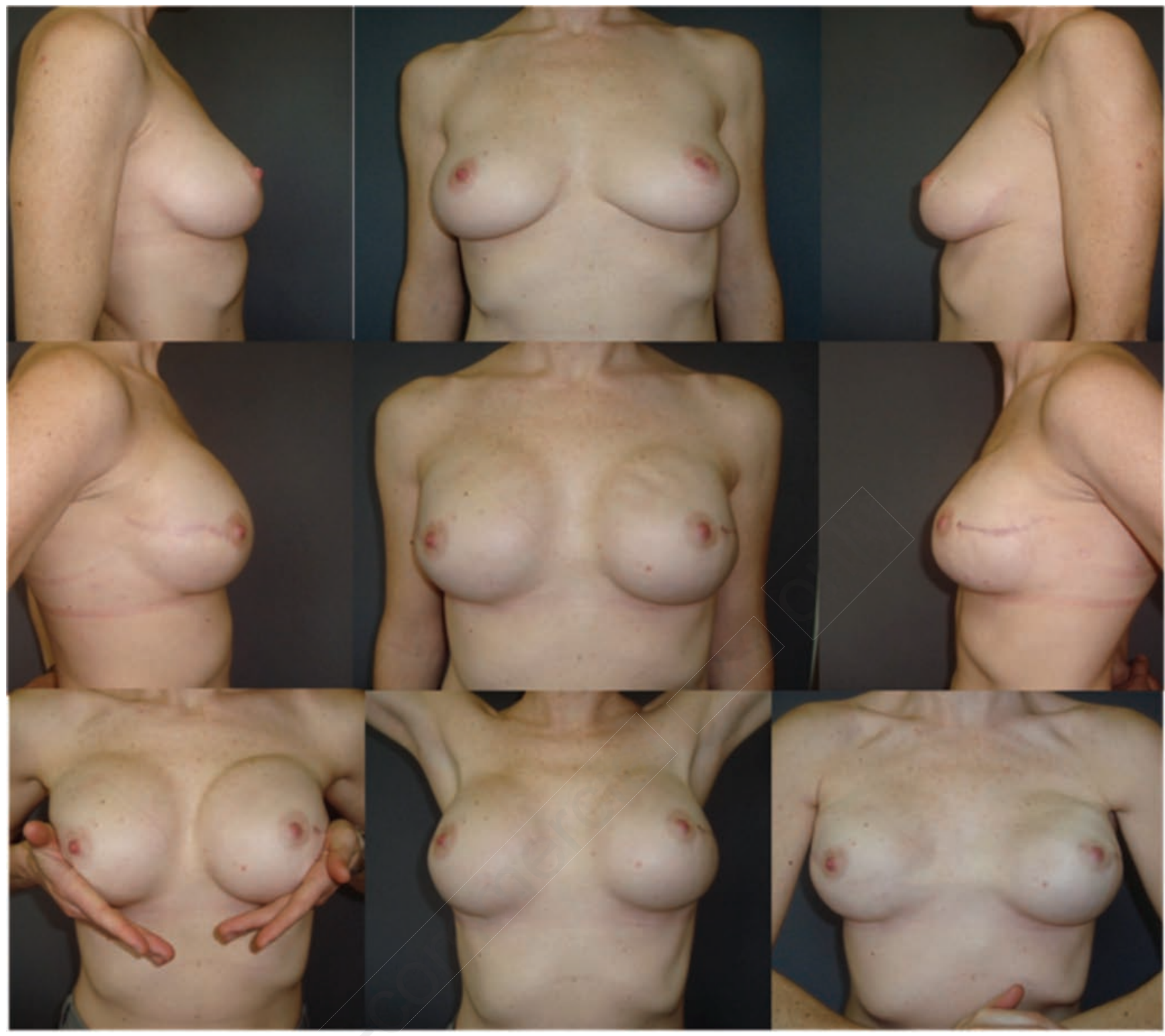

Figure 3. The patient's views: pre-operative (above); post-operative after 12 months (middle); post-operative dynamic views after 12 months (below). The absence of both capsular contracture and stiffness is almost the same. A similar behavior is detectable during active muscle tension bilaterally. Rippling appears at the left upper pole, probably due to a major looseness of the mesh pocket and decreased thickness of the pectoral muscle.

plane pocket, which is less expensive and can give similar outcome compared to the use of mesh. The development of IBR techniques using permanent implants is outstanding compared to the expensive use of ADM. The spreading use of ADM and new titanium or silk-made meshes considered as the only way to achieve the best result in breast reconstruction is mainly driven by the market.

Because the use of ADM, being derived from cadaveric human tissue, has not been approved, in several countries the market offers new biologic and synthetic products such as Strattice ${ }^{\circledR}$ and MesoBiomatrix ${ }^{\circledR}$, obtained respectively from porcine dermis and mesothelium, and others like Tiloop ${ }^{\circledR}$ or Seriscaffold $^{\circledR}$. The Tiloop ${ }^{\circledR}$ Bra (GfE Medizintechnik $\mathrm{GmbH}$ ) used in our case is a titanized polypropylene mesh made of monofilament polypropylene fibers and laser-cut edges, characterized by being extra-light and having high stretch capacity. It can minimize the shrinkage with tissue-like flexibility and high biological compatibility. On the other hand, we know that ADM and similar meshes carry their own complications. ${ }^{1}$

In the dual-plane technique, the implant is enclosed in an uninterrupted and well-vascularized, partially submuscular, pocket. This differs from other previous techniques. ${ }^{10}$ The muscle function is conserved in the upper half and the implant can expand better to lower pole, due to the high-cohesiveness of the truform ${ }^{\text {TM }}-3$ silicone and the anatomical stable shape, thus producing results similar to those with meshes. This technique also creates a lower internal bra formed by subcutaneous and fascial layers, which results in a layer thicker than simple ADM and skin. Moreover, this surgical approach is less expensive. The results obtained in our patient showed that the device dots were more palpable in mesh-based reconstruction. This could be explained by the fact that this mesh is looser or because the minimal capsular retraction causes rippling. 


\section{Conclusions}

The closed dual-plane pocket, with a continuous vascular framework embracing the breast implant, can represent a natural procedure to achieve excellent results in terms of cosmesis. ${ }^{11}$ Women receiving breast reconstruction with this technique have a high degree of satisfaction both for cosmetic and psychological reasons, and reconstruction failure is rare. ${ }^{7}$ This technique does not increase short-term local complications and spares the costs of the mesh. For these reasons, in our department closed dual-plane pocket represents the first option in selected patients, whilst meshes are mainly delegate for trials or studies. A randomized controlled trial comparing the two techniques could give an evidence-based support to the present study.

\section{References}

1. Ho G, Nguyen TJ, Shahabi A, et al. A systemic review and meta-analysis of complications associated with acellular dermal matrix-assisted breast reconstruction. Ann Plas Surg 2012;68:345-56.

2. Singh N, Reaven NJ, Funk SE. Immediate 1-stage vs. tissue expander postmastectomy implant breast reconstructions: a retrospective real-world comparison over 18 months. J Plast Reconstr Aes 2012;65:91723.

3. Cassileth L, Kohanzadeh S, Amersi F. Onestage immediate breast reconstruction with implants: a new option for immediate reconstruction. Ann Plas Surg 2012;69: 134-8.

4. Sbitany H, Sandeen SN, Amalfi AN, et al. Acellular dermis-assisted prosthetic breast reconstruction versus complete submuscular coverage: a head to head comparison of outcomes. Plast Reconstr Surg 2009; 124:1735-40.

5. Spillane CA, Ashikari AY, Koch RM, Chabner-Thompson E. An 8-year experience of direct-to-implant immediate breast reconstruction using human acellular dermal matrix (Alloderm). Breast 2010;127:514-23.

6. De Blacam C, Momoh A0, Colakoglu S, et al. Cost analysis of implant-based breast reconstruction with acellular dermal matrix. Ann Plas Surg 2012;69:516-20.

7. Riggio E, Ottolenghi J, Nava M. Breast operative technique for single-stage reconstruction after conservative skin sparing mastectomies: a preliminary study. Surg Tech Dev 2012;2:4-9.

8. Nava M, Quattrone P, Riggio E. Focus on the breast fascial system: a new approach for inframammary fold reconstruction. Plast Reconstr Surg 1998;102:1034-45.

9. Riggio E, Quattrone P, Nava M. Anatomical study of the breast superficial fascial system: the inframammary fold unit. Eur $\mathrm{J}$ Plast Surg 2000;23:310-5.

10. Salgarello M, Farallo E. Immediate breast reconstruction with definitive anatomical implants after skin-sparing mastectomy. Brit J Plast Surg 2005;58:216-22.

11. Riggio E, Nava MB. Implant-based dualplane reconstruction of the breast following sparing mastectomy. In: Spiegel AJ, ed. Breast reconstruction. Current perspectives and state of the art techniques. New York, NY: InTech Publ.; 2013. Available from: http://www.intechopen. com/books/ breast-reconstruction-current-perspectives-and-state-of-the-arttechniques/ implant-based-dual-planereconstruction-of-the-breast-followingsparing-mastectomy 